\title{
Los valores y estilos de vida de los jóvenes como factores de influencia en el consumo de alimentos internacionales
}

\section{Values and Lifestyles in Youngsters as Influence Factors when Consuming International Food}

\author{
Lizbeth Salgado Beltrán (México) \\ Licenciada en Comercio Exterior por la Universidad \\ Autónoma de Baja California Sur (México), doctora \\ en Estudios Empresariales con especialización \\ en Investigación y Técnicas de Mercado por \\ la Universidad de Barcelona (España). Correo \\ electrónico: Isalgado@caborca.uson.mx
}

\section{Dena María Jesús Camarena Gómez}

(México)

Licenciada en Economía por la Universidad de Sonora

(México), M. C. en Marketing Agroalimentario por el Instituto Agronómico Mediterráneo de Zaragoza y doctora en Economía y Gestión de las Organizaciones

por la Universidad Pública de Navarra (España).

Profesora-investigadora Asociada de tiempo completo, División de Ciencias Económicas y Administrativas de Ia Universidad de Sonora, Unidad Regional Centro. Correo electrónico: dena.camarena@eca.uson.mx

\section{Resumen \\ El objetivo de este artículo es identificar si los factores psicográficos, como los valores y estilos de vida, influyen en las actitudes de los consumidores jóvenes de alimentos internacionales. Para}

\begin{abstract}
This article aims to identify if psychographic factors, such as life values and lifestyles, affect the attitude of young people who consume international food. For this reason, we carried out univariate
\end{abstract}

\begin{tabular}{|c|c|c|}
\hline $\begin{array}{l}\text { FECHA DE RECEPCIÓN: } \\
\text { FECHA DE REVISIÓN: } \\
\text { APROBACIÓN: }\end{array}$ & $\begin{array}{r}12 \text { DE SEPTIEMBRE DE } 2014 \\
6 \text { DE OCTUBRE DE } 2014 \\
10 \text { DE OCTUBRE DE } 2014\end{array}$ & $\begin{array}{l}\text { PARA CITAR ESTE ARtículo / to CITE tHIS ARTICle } \\
\text { Salgado Beltrán, L. y Camarena Gómez, D. M. J. } \\
\text { (2014). Los valores y estilos de vida de los jóvenes } \\
\text { como factores de influencia en el consumo de alimen- } \\
\text { tos internacionales. Poliantea, 10(19), pp. 147-166. }\end{array}$ \\
\hline
\end{tabular}


ello, se realizan análisis univariantes y multivariantes, a partir de los cuales se determina el perfil psicográfico del consumidor joven. Los principales hallazgos muestran una segmentación basada en dos grupos, los denominados autoorientación, que está compuesto de dos valores y estilos de vida denominados los experimentadores y hacedores, y los luchadores e innovadores. Asimismo, tomando como referencia la escala de VALS, se encontró que la influencia de diferencias culturales es alta.

Palabras clave: escala de valores y estilos de vida, consumidor sonorense, análisis multivariantes, segmentación. and multivariate analyses in order to determine the psychographic profile of a young consumer. The main findings show a segmentation based on two groups: self-orientation -that is made by two life values and lifestyles called experimenter and doer- and fighters and innovators. Likewise, we found, taking as a reference the VALS scale, that the influence of the cultural difference is high.

Keywords: Lifestyle and value scale, consumer, multi-variable analysis, segmentation. 


\section{Los valores y estilos de vida de los jóvenes como factores de influencia en el consumo de alimentos internacionales}

\section{Values and Lifestyles in Youngsters as Influence Factors when Consuming International Food}

Lisbeth Salgado Beltrán (México)

Universidad de Sonora
Dena María Jesús Camarena Gómez

(México)

Universidad de Sonora

\section{Introducción}

Diversos estudios, fundamentalmente desarrollados en el contexto internacional, investigan los patrones alimenticios de consumidores y en qué medida estos se ven condicionados o modificados en función de las variables demográficas (nivel socioeconómico, edad, grado educativo, situación laboral y composición del hogar, entre otros) (Rivas y Grande, 2004).

Sin embargo, en años recientes, se ha observado una evolución en la segmentación del consumidor, dando paso a criterios orientados a la forma de ser y comportarse de las personas, tales como las características psicográficas. En este sentido, los valores y los estilos de vida han demostrado tener una relevancia significativa, ya que facilitan y permiten la identificación de los distintos tipos de consumidores que conforman los diversos segmentos a los cuales se enfrenta la empresa. No hay que perder de vista que los mercados son heterogéneos y los empresarios difícilmente pueden orientarse a ellos en una forma única: 
En esta línea, diversas escalas han sido elaboradas con el fin de identificar los condicionantes internos de los consumidores que influyen en sus decisiones y elecciones. Es así, que la escala de VALS ha sido elaborada por Mitchell (1983) como una herramienta que permite segmentar a los consumidores en función de sus valores personales y estilos de vida. Aunque se trata de una escala ampliamente validada en la literatura, lo cierto es que ha sido cuestionada su utilización en otros países (España, Brasil, entre otros) debido a que las influencias culturales, así como los factores económicos pueden interferir en sus resultados (Fraj et al., 2004). Esta aserción deja entrever las limitaciones que pueden existir en la escala y da origen a la necesidad de realizar su validación en distintos contextos. En este sentido, en México su aplicación ha sido exigua y sólo se ha encontrado evidencia de su utilización en los compradores de vivienda con hipoteca (Ramírez, 2009).

La escala VALS busca clasificar a los consumidores en función de sus valores y estilos de vida. Los valores, de acuerdo con la definición de Rokeach (1973), se consideran como una "creencia perdurable que de un modo específico de conducta o estado final de existencia es personalmente o socialmente preferible a un opuesto modo de conducta o estado final de existencia”. Otros autores como Kamakura y Novak (1992) reconocen que "los valores son centrales a las vidas de las personas $y$, debido a su importancia fundamental, influyen en las actitudes y los comportamientos". Para Hofstede (1997) los valores son resistentes al cambio. Se observan tres orientaciones diferentes respecto de los valores. La primera se refiere a la orientación clásica que trata de identificarlos y agruparlos por su contenido. La segunda es aquella que relaciona los valores con determinadas conductas de compra y consumo o con los atributos de los productos preferidos por los individuos. La tercera es la que establece conexiones entre los valores y diversos estilos de vida con el fin de identificar los segmentos del mercado (González, 2000).

En este contexto, los valores personales y estilos de vida se han elegido como elemento de análisis porque proporcionan información de las aspiraciones y metas que las personas jóvenes evalúan como más importantes, permitiendo conocer y determinar las motivaciones subyacentes 
que influyen en sus decisiones de compra (Blackwell, Miniard y Engel, 2002; Kahle, Gregory y Shoham, 2000; Clawson y Vinson, 1978). De ahí que en esta investigación se buscan utilizar los factores psicográficos, como los valores y estilos de vida, a fin de segmentar a los consumidores jóvenes de alimentos internacionales del noroeste de México, para lo cual se hace uso de la escala VALS.

\section{Marco teórico}

En la literatura se han encontrado varios estudios donde las principales variables utilizadas para segmentar consumidores son de tipo sociodemográfico (Della, DeJoy y Lance, 2009). Si bien este tipo de variables permiten describir a los individuos con resultados interesantes, no permiten explicar su comportamiento de compra. De ahí que las condicionantes internas, como los valores y estilos de vida, sean una herramienta eficaz para evaluar las elecciones y preferencias de los jóvenes. En este sentido, diversos estudios se han realizado considerando el poder explicativo de estas características inherentes a los individuos. A continuación, se realiza una descripción de los trabajos más relevantes en esta área.

\section{Valores y estillos de vida}

La investigación en el ámbito de los valores personales desde inicios del siglo pasado ha sido abundante. Se trata de estudios que intentar clasificar rasgos que son útiles para estudiar y entender las diferencias individuales (Eysenck, 1967; Cattell y Tregaskis, 1965). Las metodologías empleadas para este análisis son diversas; sin embargo, pese a ello se ha llegado a la conclusión de que los valores son elementos que influyen significativamente en el comportamiento de las personas (Beatty, Kahle, Homer y Misra, 1985; Yankelovich, 1981; Williams, 1979; Rokeach, 1973).

El primer uso de la palabra estilo de vida aparece en un ensayo de Max Weber de 1915, quien lo definió como la representación de características de grupos sociales quienes otorgan un estatus positivo o un "honor social" (prestigio) (Weber, 1946). Después Adler (1929), citado en Loudon y Della (1995), lo conceptualiza como aquellas metas que los individuos se imponen para así y los medios que utilizan para alcanzarlas. Según Boyd y Levy (1967), el estilo de vida se divide en dos características, primero, es compartido y segundo, se predicen características sociales homogéneas (por ejemplo los patrones de consumo iguales). 
El estilo de vida ha sido asociado al campo del marketing principalmente a la publicidad, pues esta no conseguía el efecto deseado en el público consumidor, ya que aquel no era homogéneo (Corraliza y Martín, 2000). Entonces surge la necesidad de estudiar la conducta de consumo, por lo que se considera el estilo de vida como un conjunto de valores jerarquizados y consistentes (Boyd y Levy, 1967) que pueden predecir características como los patrones de consumo. En resumen, el estilo de vida es la forma personal en la que los individuos desarrollan comportamientos y actitudes que adoptan para satisfacer sus necesidades como seres humanos y alcanzar su desarrollo personal. Para ello, se utilizó la escala de valores y estilos de vida (VALS). Se trata de una encuesta internacional elaborada por Mitchell (1983) e investigadores de SRI International ahora Strategic Business Insight (SBI), basada en la jerarquía de necesidades de Maslow (1970).

Esta escala ha sido una de las más utilizadas en la literatura sobre valores y estilos de vida. Algunos de los estudios que han aplicado la escala VALS han encontrado que presenta algunas ventajas con respecto a otras formas de medición de valores
(Kahle, Beatty y Homer, 1986), sus variables son capaces de revelar más que las demográficas y además constituye una herramienta útil para entender el comportamiento de los individuos (Shih, 1986).

Sin embargo, no hay que perder de vista que se han desarrollado diversas metodologías para medir los valores y estilos de vida, tal y como sucedió con el estudio de Kahle, Beatty y Homer (1986), en el cual se desarrolló la escala The List of Values (LOV) como alternativa de la escala VALS. Si bien la escala de LOV es más breve y sencilla de responder, lo cierto es que en algunos estudios se encontró que la escala VALS presentaba ciertas ventajas, ya que, a través de sentencias, los individuos se identificaban más fácilmente que a través de adjetivos. No obstante, la debilidad principal de este método es que no existe una forma única de aplicación, la cual resulta compleja y algunas veces confusa (Ramírez, 2009). Además, las influencias culturales de otros países pueden interferir en sus resultados, ya que tanto el número de sus dimensiones como el número de ítems que la forman pueden verse modificados (Grande, 2000). 


\section{Materiales y métodos}

Se revisó ampliamente la literatura respecto de estudios que han utilizado la escala de VALS. Se trata de un método pionero en la aplicación de la psicografía a la gestión empresarial y a la investigación de la sociedad. Actualmente este sistema clasifica a la población en ocho subgrupos (tabla 1) y establece en términos generales que las personas se agrupan en dos dimensiones: 1) la primera dimensión denominada autoorientación se divide en tres orientaciones básicas de consumo: los consumidores orientados por principios que compran tomando en cuenta "cómo debe ser" el mundo; los consumidores orientados por el estatus que basan sus compras en las opiniones y actitudes de otras personas, y los consumidores orientados a la acción que basan sus decisiones de compra en la actividad, variedad y riesgo. 2) La segunda dimensión está orientada a los recursos.

$\mathrm{El}$ instrumento está compuesto por 35 ítems que pueden responderse en una escala Likert de 4, 5 y 7 puntos. En esta investigación se evaluó a través de una escala de 5 puntos, donde 1 es totalmente en desacuerdo y 5 totalmente de acuerdo.

Tabla 1. Segmentos de la escala VALS

\begin{tabular}{|c|c|c|}
\hline \multirow{3}{*}{ Segmento } & Adjetivos & Descripción \\
\hline & \multicolumn{2}{|r|}{ 1) Por autoorientación } \\
\hline & \multicolumn{2}{|c|}{ Orientados por principios } \\
\hline $\begin{array}{l}\text { Pensadores } \\
\text { (thinkers) }\end{array}$ & $\begin{array}{l}\text { Ideales, maduros, satisfechos, } \\
\text { cómodos y reflexivos, conocedores y } \\
\text { responsables. }\end{array}$ & $\begin{array}{l}\text { Los pensadores tienen una relación moderada para las } \\
\text { instituciones de autoridad social y decoro, pero están } \\
\text { abiertos a considerar nuevas ideas. Son conservadores, los } \\
\text { consumidores prácticos, sino que buscan la durabilidad, } \\
\text { funcionalidad y valor en los productos que compran. }\end{array}$ \\
\hline $\begin{array}{l}\text { Creyentes } \\
\text { (believers) }\end{array}$ & $\begin{array}{l}\text { Idealistas, conservadores, } \\
\text { convencionales con las creencias } \\
\text { concretas sobre la base de los códigos } \\
\text { tradicionales. }\end{array}$ & $\begin{array}{l}\text { Los creyentes expresan los códigos morales que tienen } \\
\text { raíces profundas y la interpretación literal. Siguen rutinas } \\
\text { establecidas, organizadas en gran parte alrededor de } \\
\text { la casa, la familia, la comunidad y las organizaciones } \\
\text { sociales o religiosas a las que pertenecen. }\end{array}$ \\
\hline \multicolumn{3}{|c|}{ Orientados por el estatus } \\
\hline $\begin{array}{l}\text { Triunfadores } \\
\text { (achievers) }\end{array}$ & $\begin{array}{l}\text { Son motivados por el deseo de logro, } \\
\text { convencionales y son políiticamente } \\
\text { conservadores. }\end{array}$ & $\begin{array}{l}\text { Los triunfadores están activos en el mercado de consumo. } \\
\text { La imagen es importante. Debido a sus ocupadas vidas, } \\
\text { que se han interesado en una variedad de dispositivos de } \\
\text { ahorro de tiempo. }\end{array}$ \\
\hline $\begin{array}{l}\text { Sobrevivientes } \\
\text { (survivors) }\end{array}$ & $\begin{array}{l}\text { Pocos recursos, creen que el mundo } \\
\text { está cambiando demasiado rápido, se } \\
\text { sienten cómodos con lo familiar. }\end{array}$ & $\begin{array}{l}\text { Los sobrevivientes son los consumidores cautelosos. Ellos } \\
\text { representan un mercado muy modesto para la mayoría de los } \\
\text { productos y los servicios. Son leales a las marcas favoritas, } \\
\text { especialmente si pueden comprar con un descuento. }\end{array}$ \\
\hline
\end{tabular}




\begin{tabular}{|c|c|c|}
\hline \multirow{3}{*}{ Segmento } & Adjetivos & Descripción \\
\hline & \multicolumn{2}{|r|}{ 1) Por autoorientación } \\
\hline & \multicolumn{2}{|c|}{ Orientados por la acción } \\
\hline $\begin{array}{l}\text { Experimentadores } \\
\text { (experiencers) }\end{array}$ & $\begin{array}{l}\text { Libre expresión, son consumidores } \\
\text { jóvenes, entusiastas e impulsivos. } \\
\text { Experimentadores, buscan la verdad. }\end{array}$ & $\begin{array}{l}\text { Los experimentadores son ávidos consumidores y gastan } \\
\text { una proporción relativamente alta de sus ingresos en la } \\
\text { moda, el entretenimiento y la socialización. Sus compras } \\
\text { reflejan el énfasis que ponen en lucir bien y tener cosas. }\end{array}$ \\
\hline Hacedores (makers) & $\begin{array}{l}\text { Libre expresión, expresan y } \\
\text { experimentan el mundo trabajando en } \\
\text { ello, tienen habilidades constructivas y } \\
\text { autosuficiencia. }\end{array}$ & $\begin{array}{l}\text { Los fabricantes son sospechosos de nuevas ideas y } \\
\text { grandes instituciones, tales como las grandes empresas. } \\
\text { Son respetuosos de la autoridad gubernamental y los } \\
\text { trabajadores organizados, pero resentidos por la intrusión } \\
\text { del Gobierno en los derechos individuales. }\end{array}$ \\
\hline \multicolumn{3}{|c|}{ 2. Por recursos } \\
\hline $\begin{array}{l}\text { Innovadores } \\
\text { (innovators) }\end{array}$ & $\begin{array}{l}\text { Exitosos, sofisticados, alta autoestima, } \\
\text { líderes y receptivos a las nuevas ideas } \\
\text { y tecnologías. }\end{array}$ & $\begin{array}{l}\text { La imagen es importante para los innovadores no como } \\
\text { prueba de estatus o poder, sino como una expresión de } \\
\text { su gusto, la independencia y personalidad. Son uno de } \\
\text { los líderes establecidos y emergentes en los negocios y } \\
\text { el Gobierno. }\end{array}$ \\
\hline $\begin{array}{l}\text { Luchadores } \\
\text { (strivers) }\end{array}$ & $\begin{array}{l}\text { Moda, divertidos, preocupados por } \\
\text { las opiniones y la aprobación de los } \\
\text { demás, el dinero define su éxito. }\end{array}$ & $\begin{array}{l}\text { Los luchadores son consumidores activos porque las } \\
\text { compras son una actividad social y una oportunidad para } \\
\text { demostrar a sus compañeros su capacidad de compra. } \\
\text { Como consumidores, son tan impulsivos como su } \\
\text { circunstancia financiera lo permita. }\end{array}$ \\
\hline
\end{tabular}

Fuente: Modificado de http://www.strategicbusinessinsights.com

\section{Diseño muestral}

El estudio se llevó a cabo entre estudiantes universitarios en tres ciudades de la zona noroeste de México. Para el cálculo de la muestra, se tomó como referencia los datos del Instituto Nacional de Estadística y Geografía (Inegi, 2010). Posteriormente, se realizó un muestreo aleatorio estratificado, dividiendo la población de $N$ individuos, en $k$ subpoblaciones o estratos, atendiendo a criterios importantes en el estudio, que fueron la distribución de la población estudiantil universitaria en las ciudades de H. Caborca, Nogales y Hermosillo ${ }^{1}$, de tamaños respectivos $N_{1}$, ..., Nk

La asignación es proporcional al tamaño de la muestra y al tamaño del

\footnotetext{
Las tres ciudades se ubican en el noroeste de México específicamente en el estado de Sonora, donde existen registrados 82.329 estudiantes en el nivel de licenciatura (Sistema Educativo de los Estados Unidos Mexicanos, principales cifras, 2012). En la ciudad de Hermosillo la población universitaria es de 43.457 estudiantes, en Nogales y Caborca de 4.675 y 1.705 alumnos, respectivamente (Estadísticas Educativas SEC 2010-2011, 2012).
} 
estrato correspondiente con respecto a la población total (tabla 2).

Tabla 2. Ficha técnica

\begin{tabular}{|l|l|}
\hline Población & Estudiantes universitarios \\
\hline Universo & $\begin{array}{l}82329 \text { estudiantes universitarios } \\
\text { (Estadísticas Educativas SEC 2010- } \\
2011,2012)\end{array}$ \\
\hline Nivel de confianza & $95 \%$ \\
\hline $\begin{array}{l}\text { Margen de posible } \\
\text { de error }\end{array}$ & de $\pm 5 \%$ \\
\hline Muestra & 377 \\
\hline Estrato 1 Nogales & 82 \\
\hline Estrato 2 Caborca & 30 \\
\hline Estrato 3 Hermosillo & 265 \\
\hline $\begin{array}{l}\text { Fecha de recogida } \\
\text { de datos }\end{array}$ & Mayo de 2012 \\
\hline
\end{tabular}

\section{Proceso de análisis}

Inicialmente, se calcularon estadísticos univariantes para estudiar el comportamiento de las variables en forma individual al tener una primera impresión de la tendencia de los resultados (Pedret, Sagnier y Camp, 2003). Posteriormente, se realizaron varios análisis factoriales a la escala VALS, para depurar la escala y agrupar los ítems en varios factores. Es decir, se estudiaron las relaciones de interdependencia que se producen entre un conjunto de variables o individuos.

Después, se realizó el análisis de consistencia interna (alfa de Cronbach) de las subescalas resultantes del análisis factorial, y se obtuvieron valores de 0.863 a 0.692 , resultados similares a los conseguidos en otros estudios (Della, DeJoy y Lance, 2009). A partir de las puntuaciones factoriales obtenidas para cada individuo, se utilizaron, en una segunda etapa, como bases para segmentar por el análisis clúster o de conglomerados en las dos segmentaciones de VALS. El análisis clúster de K-medias es una herramienta diseñada para asignar casos a un número fijo de grupos (clústeres o conglomerados), cuyas características no se conocen aún pero que se basan en un conjunto de variables especificadas.

\section{Resultados}

\section{Análisis univariantes}

Los resultados obtenidos, en relación con las características sociodemográficas se muestran en la tabla 3. Así, se observa que, en el rango de los 18-24 años, se concentra la mayor proporción de la muestra, $88.1 \%$. Seguido de aquellos que se encuentran en el tramo de los 25-35 años de edad (10.3\%). La distribución por género es relativamente homogénea, $50.9 \%$ mujeres. El $91.8 \%$ son solteros y $6.1 \%$ casados, en tanto que otro estado civil es minoría. Respecto de los ingresos familiares, $51.5 \%$ estima que 
se ubican en el rango de los 2000 a los 8000 pesos mensuales. Como era de esperarse en una población estudiantil universitaria, la mayor parte de los encuestados se caracterizan por ser personas jóvenes, solteras y con un nivel de ingreso medio en sus familias.

Tabla 3. Características sociodemográficas de la muestra

\begin{tabular}{|l|c|l|c|}
\hline \multicolumn{1}{|c|}{ Características } & $\%$ & \multicolumn{1}{|c|}{ Característica } & $\%$ \\
\hline Edad & & $\begin{array}{l}\text { Nivel de ingresos } \\
\text { mensual }\end{array}$ & \\
\hline $18-24$ años & 88.1 & Menos de 2000 pesos & 8.0 \\
\hline 25-35 años & 10.3 & $\begin{array}{l}\text { Entre 2001-4000 } \\
\text { pesos }\end{array}$ & 21.5 \\
\hline 36-44 años & 1.1 & $\begin{array}{l}\text { Entre 4001-8000 } \\
\text { pesos }\end{array}$ & 30.0 \\
\hline 45-54 años & .5 & $\begin{array}{l}\text { Entre 8001-14 000 } \\
\text { pesos }\end{array}$ & 17.2 \\
\hline Estado civil & & $\begin{array}{l}\text { Entre 14 001-20 000 } \\
\text { pesos }\end{array}$ & 14.1 \\
\hline Casado & 6.1 & Más de 20 000 pesos & 9.3 \\
\hline Soltero & 91.8 & Sexo & 49.1 \\
\hline Separado & 1.1 & Femenino & 50.9 \\
\hline Unión libre & 1.1 & Masculino & \\
\hline
\end{tabular}

Para identificar las comidas nuevas, se hace alusión a las comidas internacionales, ya, que en los últimos años, distintas variedades de estas se han introducido con éxito en el mercado nacional. En este sentido, se puede observar en la tabla 5 que el consumo de este tipo de comidas entre los encuestados es relativamente alto, ya que en $88.3 \%$ de los casos las han degustado en los últimos seis meses. Incluso puede considerarse que, además de conocer estas comidas, las consumen de manera frecuente, ya que $62.8 \%$ las degusta con una frecuencia igual o superior a una vez cada 15 días.

Tabla 4. Conocimiento y consumo de comidas internacionales

\begin{tabular}{|l|c|}
\hline \multicolumn{1}{|c|}{ Conocimiento y consumo } & $\%$ \\
\hline $\begin{array}{l}\text { Si ha consumido comida internacional en los últimos } \\
\text { seis meses }\end{array}$ & 88.3 \\
\hline $\begin{array}{l}\text { No ha consumido comida internacional en los últimos } \\
\text { seis meses }\end{array}$ & 10.3 \\
\hline Consume comidas internacionales diariamente & 1.5 \\
\hline $\begin{array}{l}\text { Consume comidas internacionales una vez por } \\
\text { semana }\end{array}$ & 34.5 \\
\hline $\begin{array}{l}\text { Consume comidas internacionales una vez cada } \\
\text { 15 días }\end{array}$ & 26.8 \\
\hline Consume comidas internacionales una vez al mes & 33.0 \\
\hline Consume comidas internacionales con otra frecuencia & 4.2 \\
\hline
\end{tabular}

Respecto de las comidas internacionales que los estudiantes universitarios han probado, destaca con $24.5 \%$ de consumidores la comida china, seguida de la estadounidense con $19.3 \%$. Otras opciones, como la italiana y japonesa, también han sido degustadas por un porcentaje significativo de consumidores, 17.4 y $14.1 \%$, respectivamente. En tanto que, entre las opciones con menor consumo, se encuentran la árabe (1.9\%), argentina (1.2\%) y francesa (5.0\%) (tabla 5). 
Tabla 5. Comidas

internacionales consumidas

\begin{tabular}{|l|c|l|c|}
\hline \multicolumn{1}{|c|}{ Comidas } & $\%$ & \multicolumn{1}{c|}{ Comidas } & $\%$ \\
\hline China & 24.5 & Española & 5.2 \\
\hline Estadounidense & 19.3 & Francesa & 5.0 \\
\hline Italiana & 17.4 & Otras asiáticas & 2.7 \\
\hline Japonesa & 14.1 & Argentina & 1.2 \\
\hline Latinoamericana & 8.7 & Árabes & 1.9 \\
\hline
\end{tabular}

En relación con el lugar de adquisición de las comidas internacionales, la mayor proporción de los consumidores la compra en los restaurantes (59.7\%), seguido de la tienda especializada (14.4\%). Otras opciones tienen una menor frecuencia de compra, los supermercados. Estos resultados no son extraños si se considera que el conocimiento sobre la forma de elaboración aún no se encuentra muy generalizado, sobre todo porque se trata de comidas que no forman parte de la dieta habitual de los consumidores.

\section{Análisis multivariantes Análisis factorial}

En una primera fase, tras la realización de varios análisis factoriales, la prueba de esfericidad de Bartlett fue significativo y el KMO (Kaiser-Meyer-Olkin) obtuvo el valor de 0.817 , con lo cual se comprobó que existe suficiente correlación entre las variables para llevar a cabo el análisis factorial en la muestra de consumidores. Se utilizó el método de extracción de componentes principales con rotación varimax, seleccionando el número de factores mediante el criterio del valor propio. Los resultados muestran que de los 35 ítems que consta la escala VALS, se eliminaron 13 , ya que su contribución era inferior a 0.5 (tabla 6) en los factores.

Los valores se agruparon en siete valores y estilos de vida que explican en $63.47 \%$ de la varianza y que seis de ellos pertenecen a la escala original VALS: experimentadores, luchadores, hacedores, innovadores, pensadores $y$ creyentes. El factor denominado conformistas se creó a partir de los ítems agrupados. Si bien la escala original está dividida en ocho subgrupos, se deduce, primero, que el estudio se realizó entre jóvenes universitarios, lo cual generó una variación entre los segmentos; y segundo, la influencia de las diferencias culturales entre países se ve reflejada en los nuevos grupos conformados. 
Tabla 6. Análisis factorial de los valores y estilos de vida VALS

\begin{tabular}{|c|c|c|c|c|c|c|c|c|}
\hline VALS & Experimentadores & Luchadores & Hacedores & Innovadores & Conformistas & Pensadores & Creyentes & Comunalidad \\
\hline $\begin{array}{l}\text { Me gusta hacer cosas } \\
\text { que son nuevas y } \\
\text { diferentes. }\end{array}$ & 0.796 & -0.031 & 0.022 & 0.124 & -0.073 & 0.121 & 0.095 & 0.679 \\
\hline $\begin{array}{l}\text { Me gusta el reto de } \\
\text { hacer algo que nunca } \\
\text { he hecho antes. }\end{array}$ & 0.785 & -0.008 & -0.113 & 0.15 & -0.026 & 0.001 & 0.06 & 0.656 \\
\hline $\begin{array}{l}\text { Me gusta aprender } \\
\text { cosas, aunque nunca } \\
\text { sean de alguna } \\
\text { utilidad para mí. }\end{array}$ & 0.733 & -0.042 & 0.046 & -0.006 & 0.006 & 0.191 & 0.03 & 0.579 \\
\hline $\begin{array}{l}\text { Estoy siempre } \\
\text { en busca de una } \\
\text { emoción. }\end{array}$ & 0.726 & 0.069 & 0.125 & 0.062 & -0.104 & 0.212 & 0.087 & 0.614 \\
\hline $\begin{array}{l}\text { Me gusta hacer cosas } \\
\text { con mis manos. }\end{array}$ & 0.699 & -0.083 & 0.206 & 0.085 & 0.058 & 0.08 & -0.007 & 0.555 \\
\hline $\begin{array}{l}\text { Me gusta que haya } \\
\text { variedad en mi vida. }\end{array}$ & 0.661 & -0.078 & -0.155 & 0.003 & -0.097 & 0.376 & 0.11 & 0.630 \\
\hline $\begin{array}{l}\text { Me gusta ser } \\
\text { considerado como } \\
\text { una persona que está } \\
\text { a la moda. }\end{array}$ & 0.046 & 0.809 & 0.087 & 0.044 & 0.065 & -0.107 & 0.02 & 0.682 \\
\hline $\begin{array}{l}\text { Sigo las últimas } \\
\text { tendencias y modas. }\end{array}$ & -0.011 & 0.794 & -0.051 & -0.004 & 0.037 & -0.008 & 0.058 & 0.637 \\
\hline $\begin{array}{l}\text { Me gusta ir vestido a } \\
\text { la moda. }\end{array}$ & -0.056 & 0.793 & 0.045 & 0.055 & -0.117 & 0.042 & -0.028 & 0.654 \\
\hline $\begin{array}{l}\text { Me gusta ir a la } \\
\text { moda más que al } \\
\text { resto de la gente. }\end{array}$ & -0.101 & 0.768 & 0.031 & 0.061 & 0.15 & -0.044 & 0.091 & 0.638 \\
\hline $\begin{array}{l}\text { Me gusta mirar } \\
\text { a través de } \\
\text { restaurantes y } \\
\text { tiendas de alimentos. }\end{array}$ & 0.011 & 0.017 & 0.838 & 0.081 & 0.069 & -0.046 & -0.01 & 0.716 \\
\hline $\begin{array}{l}\text { Me interesa la } \\
\text { mecánica, los } \\
\text { motores y el trabajo. }\end{array}$ & 0.048 & -0.044 & 0.783 & 0.027 & 0.069 & 0.104 & 0.159 & 0.658 \\
\hline $\begin{array}{l}\text { Me gusta hacer cosas } \\
\text { de madera, metal y } \\
\text { otros materiales. }\end{array}$ & 0.032 & 0.139 & 0.762 & 0.007 & 0.077 & 0.042 & -0.082 & 0.615 \\
\hline $\begin{array}{l}\text { Me gusta dirigir } \\
\text { a otros. }\end{array}$ & 0.155 & 0.037 & 0.041 & 0.854 & 0.009 & 0.056 & 0.052 & 0.763 \\
\hline $\begin{array}{l}\text { Me gusta estar a } \\
\text { cargo de un grupo. }\end{array}$ & 0.172 & 0.077 & 0.04 & 0.762 & -0.078 & -0.073 & 0.176 & 0.660 \\
\hline $\begin{array}{l}\text { En realidad solo } \\
\text { estoy interesado en } \\
\text { algunas cosas. }\end{array}$ & 0.031 & 0.022 & 0.054 & 0.06 & 0.834 & -0.042 & -0.139 & 0.725 \\
\hline $\begin{array}{l}\text { Admito que me } \\
\text { interesan pocas } \\
\text { cosas. }\end{array}$ & -0.175 & 0.076 & 0.141 & -0.119 & 0.770 & -0.033 & 0.09 & 0.673 \\
\hline
\end{tabular}


Tabla 6. Análisis factorial de los valores y estilos de vida VALS

\begin{tabular}{|c|c|c|c|c|c|c|c|c|}
\hline VALS & Experimentadores & Luchadores & Hacedores & Innovadores & Conformistas & Pensadores & Creyentes & Comunalidad \\
\hline $\begin{array}{l}\text { Me gusta la } \\
\text { gente y las cosas } \\
\text { extravagantes. }\end{array}$ & 0.36 & 0.08 & 0.134 & -0.083 & -0.056 & 0.707 & 0.004 & 0.665 \\
\hline $\begin{array}{l}\text { En ocasiones estoy } \\
\text { interesado en nuevas } \\
\text { teorías. }\end{array}$ & 0.297 & -0.185 & -0.007 & 0.113 & 0.002 & 0.697 & 0.062 & 0.624 \\
\hline $\begin{array}{l}\text { Hoy en día hay } \\
\text { mucho sexo en la TV. }\end{array}$ & 0.319 & -0.039 & -0.132 & -0.015 & 0.035 & -0.066 & 0.656 & 0.557 \\
\hline $\begin{array}{l}\text { Me considero una } \\
\text { persona intelectual. }\end{array}$ & 0.15 & 0.023 & 0.089 & 0.304 & -0.116 & 0.227 & 0.594 & 0.540 \\
\hline $\begin{array}{l}\text { El Gobierno } \\
\text { debería fomentar } \\
\text { la enseñanza de } \\
\text { la religión en las } \\
\text { escuelas públicas. }\end{array}$ & -0.31 & 0.309 & 0.237 & 0.06 & 0.002 & -0.008 & 0.567 & 0.573 \\
\hline Varianza (\%) & $20.24 \%$ & $13.8 \%$ & $8.81 \%$ & $6.26 \%$ & $5.25 \%$ & $4.62 \%$ & $4.38 \%$ & \\
\hline \multicolumn{9}{|c|}{ Total de varianza $63.47 \%$} \\
\hline \multirow{2}{*}{\multicolumn{3}{|c|}{$\begin{array}{l}\text { Medida de adecuación muestral de Kaiser-Meyer-Olkin (KMO): } \\
\text { Prueba de esfericidad de Bartlett: }\end{array}$}} & & & \multicolumn{4}{|c|}{0.817} \\
\hline & & & \multicolumn{6}{|c|}{2877938 (valor p 0.000) } \\
\hline
\end{tabular}

En el análisis de consistencia significativamente por encima de interna (alfa de Cronbach) cuatro los límites inferiores aceptables, tal de las siete subescalas fueron va- y como mencionan Nunnally (1967) lidadas (tabla 7) con coeficientes y Hair et al. (2005).

Tabla 7. Análisis de consistencia de las subescalas

\begin{tabular}{|c|c|c|c|c|}
\cline { 2 - 5 } \multicolumn{1}{c|}{} & \multicolumn{2}{c|}{ Por autoorientación } & \multicolumn{2}{c|}{ Por recursos } \\
\cline { 2 - 5 } \multicolumn{1}{c|}{ Orientados por la acción } & & \\
\hline \multirow{2}{*}{ Subescalas } & Experimentadores & Hacedores & Luchadores & Innovadores \\
\hline $\boldsymbol{\alpha}$ & 0.863 & 0.744 & 0.816 & 0.692 \\
\hline
\end{tabular}




\section{Anólisis clúster k-medias}

Las puntuaciones factoriales obtenidas para cada individuo se utilizaron en una segunda fase como bases para segmentar por el análisis clúster o de conglomerados por el método de reasignación (k-means). Con este procedimiento, se encontraron dos segmentos en el grupo de consumidores jóvenes (tabla 9). El primero (por autoorientación) presenta un valor positivo en los factores "experimentadores" y "hacedores". Los "experimentadores" están formados por personas que se caracterizan por la libre expresión, consumidores jóvenes ( $88.2 \%$ en edad de 18 a 24 años), entusiastas e impulsivos que buscan variedad y emociones. Los "hacedores", además de caracterizarse por la libre expresión, expresan y experimentan el mundo trabajando en ello, tienen habilidades constructivas y valor autosuficiencia. Es decir, son jóvenes orientados a la acción.

En el segundo segmento, se encuentran aquellas personas que valoran más los recursos. Los "luchadores” están interesados en la moda, son divertidos, preocupados por las opiniones y la aprobación de los demás; el dinero define su éxito. Los "innovadores" son jóvenes exitosos, sofisticados, con alta autoestima, líderes y receptivos a las nuevas ideas y tecnologías. Así, los dos segmentos tienen tamaños ligeramente distintos, segmento por autoorientación $58.60 \%$ y por recursos $41.40 \%$.

Tabla 9. Segmentación de los consumidores en función de sus valores y estilos de vida

\begin{tabular}{|c|c|c|}
\hline \multirow{2}{*}{ Factores } & \multicolumn{2}{|c|}{ Conglomerado de pertenencia } \\
\cline { 2 - 3 } & Por autoorientación & \multirow{2}{*}{ Por recurso } \\
\cline { 2 - 3 } & Orientados a la acción & \\
\hline Experimentadores & 0.4401 & -0.62348 \\
\hline Luchadores & -0.4185 & 0.59288 \\
\hline Hacedores & 0.24067 & -0.34095 \\
\hline Innovadores & -0.11736 & 0.16627 \\
\hline \% de personas & $58.60 \%$ & $41.40 \%$ \\
\hline
\end{tabular}

De acuerdo con la frecuencia de consumo de alimentos internacionales de estos segmentos, los autoorientados (34.70\%) consumen más al mes que el segmento por recursos (30.7\%) (tabla 10). 
Tabla 10. Frecuencia consumo de comidas internacionales

\begin{tabular}{|c|c|c|c|c|c|}
\hline Segmentos & Diariamente & $\begin{array}{c}\text { Una vez por } \\
\text { semana }\end{array}$ & $\begin{array}{c}\text { Una vez cada } \\
15 \text { dias }\end{array}$ & Una vez al mes & Otro \\
\hline Por autoorientación & 3 & 65 & 52 & 68 & 8 \\
\hline$(n=221)$ & $1.50 \%$ & $33.20 \%$ & $26.50 \%$ & $34.70 \%$ & $4.10 \%$ \\
\hline Por recursos & 2 & 51 & 38 & 43 & 6 \\
\hline$(n=156)$ & $1.40 \%$ & $36.40 \%$ & $27.10 \%$ & $30.70 \%$ & $4.30 \%$ \\
\hline
\end{tabular}

Para contrastar si existe asocia- en ambos segmentos en 18-24 años, ción entre los distintos segmentos así como el estado civil en soltero. y las características sociodemográ- La diferencia más notable es el nivel ficas de los consumidores, se reali- de ingreso mensual familiar, los auzó la prueba de chi-cuadrado (tabla toorientados ganan más y los orien11). Ambos segmentos presentan si- tados por recursos se concentran en militudes en las variables; la razón los salarios bajos. Los "luchadores" de esto es que el estudio está enfoca- que pertenecen a este segmento son do en los jóvenes universitarios. Los definidos por el dinero que ganan, por consumidores autoorientados son eso siempre están buscando cómo demás hombres (52.9\%) que mujeres mostrar a los demás su capacidad de (47.1\%), y en caso contrario, en el segmento por recursos son más mujeres (56.4\%) que hombres (43.6\%). compra; eso no implica que tengan capacidad de compran, pero sí que se esfuerzan por conseguirlo.

En el caso de la edad, se concentra 
Tabla 11. Perfil sociodemográfico de los segmentos identificados en función de los valores y estilos de vida

\begin{tabular}{|c|c|c|c|}
\hline Variables & Test $X^{2}$ & Por autoorientación & Por recursos \\
\hline & Valor de prob. & $(n=221)$ & $(n=156)$ \\
\hline \multicolumn{4}{|c|}{ Características sociodemográficas } \\
\hline Sexo & $0.004^{\star \star \star}$ & & \\
\hline Hombre & & $52.90 \%$ & $43.60 \%$ \\
\hline Mujer & & $47.10 \%$ & $56.40 \%$ \\
\hline Edad & $0.009^{\star \star \star}$ & & \\
\hline 18-24 años & & $88.20 \%$ & $87.80 \%$ \\
\hline 25-35 años & & $10.00 \%$ & $10.90 \%$ \\
\hline 36-44 años & & $1.40 \%$ & $0.60 \%$ \\
\hline 45-54 años & & $0.50 \%$ & $0.60 \%$ \\
\hline Estado civil & $0.008^{\star \star \star}$ & & \\
\hline Casado & & $6.80 \%$ & $5.10 \%$ \\
\hline Soltero & & $91.00 \%$ & $92.90 \%$ \\
\hline Divorciado & & $0.50 \%$ & $0.00 \%$ \\
\hline Separado & & $0.90 \%$ & $0.60 \%$ \\
\hline Unión libre & & $0.90 \%$ & $1.30 \%$ \\
\hline \multicolumn{4}{|c|}{ Ingreso mensual familiar } \\
\hline Menos de $\$ 2000$ & & $8.60 \%$ & $7.10 \%$ \\
\hline$\$ 2001-4000$ & & $20.40 \%$ & $23.10 \%$ \\
\hline$\$ 4001-8000$ & & $31.70 \%$ & $27.60 \%$ \\
\hline$\$ 8001-14000$ & & $16.70 \%$ & $17.90 \%$ \\
\hline$\$ 14001-20000$ & & $11.30 \%$ & $17.90 \%$ \\
\hline Mas de $\$ 20000$ & & $11.30 \%$ & $6.40 \%$ \\
\hline
\end{tabular}

Nota: Únicamente se incluyen las variables que resultaron significativamente distintas entre segmentos. Test de chi-cuadrado: *** $y$ ** indica una asociación significativa a 1 y $5 \%$, de nivel de significación, respectivamente.

\section{Discusión y recomendaciones}

Las variables psicográficas, como los valores y los estilos de vida, han demostrado que son relevantes, puesto que facilitan la identificación de los distintos tipos de consumidores que conforman los diversos segmentos ante los que se enfrenta la empresa. Diversos estudios (Rivas y Grande, 2004; Kahle, 1996) han concluido sobre la importancia de combinar las variables psicográficas con las sociodemográficas con el afán de obtener 
mejores resultados, de ahí que este estudio considere ambos criterios.

Por lo anterior, esta investigación buscó utilizar los factores psicográficos, como los valores y estilos de vida, para segmentar a los consumidores de alimentos internacionales del noroeste de México, para lo cual se hizo uso de la escala VALS.

Los principales hallazgos muestran la agrupación en dos segmentos denominados por autoorientación $(\mathrm{n}=221)$, que está compuesto de dos valores y estilos de vida denominados los experimentadores y hacedores, $\mathrm{y}$ por recursos $(\mathrm{n}=156)$ que comprende a los luchadores e innovadores. Si bien la escala VALS original comprende ocho subgrupos, en este estudio se puso de manifiesto que la influencia de las diferencias culturales es alta, ya que estos resultados son semejantes a otros estudios llevados a cabo en países de Europa (Fraj et al., 2004), Latinoamérica (Gil y Cortez, 2010) y Asia (Li y Cai, 2011), en los cuales se han modificados los valores y estilos de vida de los consumidores.

En el por autoorientación, son jóvenes orientados a la acción. Los experimentadores son considerados como jóvenes entusiastas que les gusta probar sobre lo nuevo, es decir, experimentan sobre las comidas internacionales, generalmente gastan una proporción relativamente alta de sus ingresos en la moda y entretenimiento. Los hacedores son creadores de nuevas ideas, y en el momento de trabajar son organizados; se infiere que estan abiertos a nuevas comidas, como los platillos internacionales, debido a su espíritu libre. Este segmento gana un poco más que el segmento por recursos y se encuentran más hombres que mujeres.

Por otro lado, en el segmento por recurso, los luchadores son impulsivos en las compras, les gusta dar una imagen de tener alta capacidad de compra, aunque no necesariamente la tengan. Como les preocupa la aprobación de su grupo social, están dispuestos a consumir alimentos extranjeros para quedar bien con ellos. Los innovadores son líderes y sofisticados, de buen gusto, son los más receptivos de este grupo a las nuevas ideas como nuevos negocios de comida. Este segmento tiene menos ingreso mensual familiar y está integrado en su mayoría por mujeres.

Desde el punto de vista empresarial, el uso de esta segmentación por valores y estilos de vida por parte de los mercadólogos puede implicar la generación de estrategias diferenciadoras para la comunicación y el 
desarrollo de productos, así como la creación de nuevas empresas de comidas internacionales dirigidas a los perfiles encontrados.

Hay que tener en cuenta que esta investigación es de carácter exploratorio y se circunscribe a una región específica de estudio, por lo que los resultados no son concluyentes y la extrapolación a otros entornos debe realizarse con cautela.

\section{Referencias bibliográficas}

Beatty, S., Kahle, L., Homer, P. y Misra, S. (1985). Alternative measurement approaches to consumer values: the list of values and the Rokeach value survey. Psychology \& Marketing, 2, 181-200.

Blackwell, R. D, Miniard, P. W. y Engel, J. F. (2002). Comportamiento del

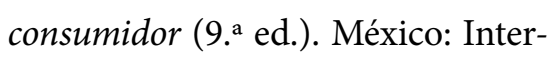
national Thomson Editores.

Boyd Jr., Harper W. y Levy, Sidney J. (1967). Promotion: a behavioral view. Foundations of marketing series. New Jersey: Prentice Hall.

Cattell, R. B. y Tregaskis, D. V. G. (1965). The scientific analysis of personality (vol. 27). Baltimore: Penguin Books.

Clawson, C. J. y Vinson, D. E. (1978). Human values: a historical and interdisciplinary analysis. Advances in Consumer Behavior, 5, 396-402.

Corraliza J. A. y Martín, R. (2000). Estilos de vida, actitudes y comportamientos ambientales. Medio Ambiente y Comportamiento Humano, 1(1), 31-56.

Della, L. J., DeJoy, D. M. y Lance, C. E. (2009). Explaining fruit and vegetable intake using a consumer marketing tool. Health Education \& Behavior, 36(5), 895-914.

Estadísticas Educativas SEC 20102011 (2012). http://168.255.106.22/ principalescifras/Default.aspx Eysenck, H. J. (1967). The biological basis of personality. Transaction Publishers.

Gobierno Federal (2012, noviembre). Sistema Educativo de los Estados Unidos Mexicanos, principales cifras, ciclo escolar 2011-2012. Recuperado de http://www.sep.gob.mx/work/models/sep1/Resource/1899/2/images/ principales_cifras_2011_2012.pdf 
González Fernández, A. M. (2000). Los valores personales en el comportamiento del consumidor. Revisión de diversas metodologías aplicadas al marketing. Esic Market, 9-36.

Hair, J.F., Black, W., Babin, B., Anderson, R.E., \& Tatham, R.L. (2005). Multivariate data analysis (5th ed.). Upper Saddle River, NJ: Prentice Hall

Hofstede, G. (1997). Cultures and organizations: software of the mind. Nuevo York: McGraw-Hill.

Kahle, L., Gregory, R. y Shoham, A. (2000). Findings of LOV throughout the world, and other evidence of Cross-national consumer psychographics: Introduction. En L. Kahle (ed.), Cross-national consumer psychographic (pp. 1-13). Nueva York: International Business Press.

Kahle, L. R. (1996). Social values and consumer behaviour: research from the list of values. En C. Seligman, J. M. Olson y M. P. Zanna (eds.), The psychology of values: The Ontario Symposium (vol. 8, pp. 135-150). Lawrence Erlbaum Associates.

Kahle, L. R., Beatty, S. E. y Homer, P. (1986). Alternative measurement approaches to consumer values: the list of values (LOV) and values and life style (VALS). Journal of Consumer Research, 13, 405-409.

Kamakura, W. A. y Novak, T. P. (1992). Value-system segmentation: exploring the meaning of LOV. Journal of Consumer Research, 19, 119132.

Li, M. y Cai, L. A. (2011). The effects of personal values on travel motivation and behavioral intention. Journal of Travel Research, 51(4), 473-487.

Loudon D. L. y Della B., Albert J. (1995). Comportamiento del consumidor: conceptos y aplicaciones, México: McGraw-Hill.

Maslow, A. (1970). A theory of human motivation. En A. Maslow (ed.), Motivation and personality (pp. 35-57). Nueva York y Londres: Harper and Row Publishers.

Mitchell, A. (1983). The nine american life styles. Nueva York: Warner.

Pedret, R., Sagnier, L. y Camp, F. (2003). Herramientas para segmentar mercados y posicionar productos: análisis de información cualitativa en investigación comercial. Barcelona: Deusto. 
Ramírez Torres, E. (2009). La segmentación por estilo de vida. Datos, diagnósticos, tendencias, 18 (2. época), año 16.

Rivas, J. A. y Grande, E. I. (2004). Comportamiento del consumidor (5. ${ }^{\mathrm{a}}$ ed.). Madrid: ESIC.

Rokeach, M. (1973). The antur of human values. Nueva York: The Free Press.

Salinas, E. M., Esteban, I. G. y Andrés, E. F. (2004). Un estudio exploratorio sobre las variables psicográficas que influyen en el comportamiento del consumidor ecológico. Revista de Economía y Empresa, 21(50), 61-88.

Shih, D. (1986). VALS as a tool of tourism market research: the pennsylvania experience. Journal of Travel Research, 24(4), 2-11.
Strategic Business Insight (SBI). Recuperado de http://www.strategicbusinessinsights.com

Weber, M. (1946). The social psychology of the world religions. En $\mathrm{H}$. H. Gerth y C. W. Mills (eds.), From Max Weber: essays in sociology. Nueva York: Oxford University Press.

Williams, R. M. (1979). Change and stability in values and value perspectives: a sociological perspective. En M. Rokeach (ed.), Understanding human values: individual and societal. Nueva York: Free Press.

Yankelovich, D. (1981). New rules: searching for self-fulfillment in a world turned upside down. New York: Bantam. 